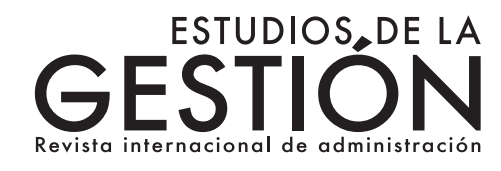

\title{
Fidelización en el sector cooperativo de carácter institucional en Ecuador
}

\author{
Loyalty in the institutional cooperative \\ sector in Ecuador
}

Fidelização no setor cooperativo de carácter institucional no Equador

\author{
Karol Zambrano \\ Universidad Andina Simón Bolívar, Sede Ecuador \\ karolzzf2@hotmail.com
}

DOI: https://doi.org/10.32719/25506641.2019.6.4

Fecha presentación: 6 de junio de 2019 • Fecha de aceptación: 19 de agosto de 2019 Artículo de investigación

Licencia Creative Commons

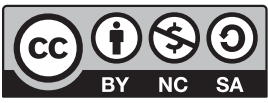




\section{Resumen}

El sector cooperativista, también denominado de la Economía Popular y Solidaria, ha presentado un crecimiento y fortalecimiento sostenido a lo largo de las últimas décadas, por lo que se vuelve una necesidad importante medir la fidelización de sus clientes como medida de control y mitigación de posibles deserciones de clientes, y también determinar la influencia del factor trabajo en la consolidación de la lealtad en los asociados. En el presente trabajo se midió la fidelización de clientes en la Cooperativa de Ahorro y Crédito Ciudad de Quito, que es una cooperativa institucional vinculada al Gobierno de los Estados Unidos. La base de datos consta de 465 observaciones y el modelo econométrico usado fue el Logit. Los principales resultados evidenciaron que la variable con mayor influencia en la fidelización de clientes es la antigüedad, seguida por el monto total de ahorros y créditos. También se determinaron variables que generan disminución en el nivel de fidelización, tales como la edad de los asociados y el número de créditos que los asociados mantienen en la entidad.

Palabras clave: Fidelización, deserción, retención.

JEL: G21 Bancos, otras instituciones de depósito; hipotecas.

\section{Abstract}

The cooperative sector, also known as the Popular and Solidarity Economy has presented sustained growth and strengthening over the last decades, thus to measure customer loyalty as a measure of control and mitigation of possible defections of clients is needed, and also to determine the influence of labor in the consolidation of loyalty in the associates. In this work, customer loyalty was measured in Ciudad de Quito Savings and Credit Cooperative, which is an institutional cooperative linked to the United States government. The database consists of 465 observations and the econometric model used was the Logit. The main results showed that the variable with the greatest influence on customer loyalty is seniority, followed by the total amount of savings and credits. Variables that generate a decrease in the level of loyalty were also determined, such as the age of the associates and the number of credits that the associates maintain in the entity.

Keywords: Loyalty, attrition, retention.

JEL: G21 Banks, Other deposit institutions, Mortgages.

\section{Resumo}

O setor cooperativista, também denominado como da Economia Popular e Solidária, apresentou um crescimento e fortalecimento sustentável ao longo das últimas décadas, pelo que se torna una necessidade importante medir a fidelização de seus clientes como medida de controle e mitigação de possíveis deserções de clientes, e também determinar a influência do fator trabalho na consolidação da lealdade nos associados. No presente trabalho foi medida a fidelização de clientes na Cooperativa de Poupança e Crédito Ciudad de Quito, que é uma cooperativa institucional vinculada ao governo dos Estados Unidos. A base de dados está constituída por 465 observações e o modelo econométrico 
usado foi o Logit. Os principais resultados evidenciaram que a variável com maior influência na fidelização de clientes é a antiguidade, seguida pela quantidade total de poupanças e créditos. Também foram determinadas variáveis que geram diminuição no nível de fidelização, tais como a idade dos associados e o número de créditos que os associados mantêm na entidade.

Palavras-chave: Fidelização, deserção, retenção.

JEL: G21 Bancos, outras instituições de depósito, hipotecas.

\section{Introducción}

L

a influencia de la economía popular y solidaria (EPS) en la generación de empleo ha sido un factor importante para Ecuador; así, en 2013 la exministra de Inclusión Económica y Social, Doris Soliz, señaló: “La economía popular y solidaria genera el $60 \%$ de total de empleo en Ecuador. El $55 \%$ del empleo en el área urbana es generado por la economía popular y solidaria. De igual manera, un $71 \%$ del emprendimiento económico popular se desarrolla en las zonas rurales mediante la agricultura, ganadería, caza y silvicultura, y finalmente existen otras actividades como la manufactura, turismo, entre otras, que aportan con un 29\%" (MIES 2013, párr. 2).

En este contexto, el sector cooperativista se ha ido fortaleciendo con el tiempo, convirtiéndose en un sector capaz de competir con los bancos más grandes a nivel nacional. Este componente del sector financiero del país es la forma de organización empresarial más conocida de la economía social y solidaria (SEPS 2014), y tiene como esencia el crecimiento socioeconómico de sus asociados, para lo cual se basa en siete principios cooperativos que tienen como objeto evidenciar esta parte social y democrática que caracteriza a las instituciones que forman parte de la EPS, la preocupación por la comunidad, el generar un crecimiento profesional del personal que forma parte de estas instituciones, al igual que concienciar que los socios no son únicamente usuarios, sino actores clave del proceso de desarrollo y fortalecimiento de estas organizaciones al ser propietarios de las mismas.

Se han emitido un sinnúmero de controles, tales como la emisión del Código Orgánico Financiero y Monetario en 2014, la Ley Orgánica de Economía Popular y Solidaria (LOEPS) y su respectivo reglamento, Resoluciones de la Superintendencia de Economía Popular y Solidaria (SEPS) y 
varios documentos de la Junta de Política y Regulación Monetaria y Financiera desde el año 2014. Este hecho ha provocado que muchas instituciones de la EPS se liquiden o se absorban; el número de cooperativas de ahorro y crédito (COAC) que siguen funcionando en el país es significativo. Según el Boletín Financiero emitido por la SEPS, en marzo de 2019 existían un total de 593 COAC divididas en diferentes segmentos, que alcanzan montos por USD 14 mil millones en activos, USD 12 mil millones en pasivos y aproximadamente USD 2 mil millones en patrimonio.

Es preciso destacar que, si bien el Código Orgánico Monetario y Financiero categorizó a las COAC por el número de activos (EC 2014, art. 447), existe otra categoría en la que deberían segmentarse, y estas son las de naturaleza abierta y las de carácter institucional, es decir, aquellas que no tienen acceso al público en general, y se consolidan con grupos específicos de asociados. En el presente trabajo se trata específicamente de un segmento denominado de carácter institucional. Si bien sería importante definir cuántas cooperativas son parte de esta categoría, no existen datos públicos disponibles, convirtiéndose en un aspecto que consta únicamente en el estatuto interno de las cooperativas.

Considerando el entorno del cooperativismo en Ecuador, la investigación tiene como objetivo principal analizar el impacto de variables discretas en la probabilidad de fidelidad de un asociado en las COAC de carácter institucional. De esta manera, es importante entender el concepto de fidelización dentro del contexto de este trabajo.

Una de las principales características de la fidelización es el aporte del capital de trabajo como factor clave para la consecución del mismo. Al concentrarse netamente en la fidelización del cliente, no en una marca o en un determinado producto, se vuelve evidente que el factor trabajo es un componente clave a la hora de lograr una real fidelización o lealtad de un cliente a un determinado servicio o producto.

De acuerdo con Uncles, Dowling y Hammond (2003), la lealtad de un cliente a diferentes aspectos como la marca, un determinado servicio o un producto específico, es un hecho cotidiano; sin embargo, se ha catalogado únicamente como lealtad de marca, cuando lo correcto sería enfatizar en que la lealtad es una característica propia del cliente; también se hace alusión a que no existe una definición universalmente aceptada, por lo que se hace 
necesaria una aproximación. Se plantean escenarios específicos: la lealtad como una actitud que a veces conduce a una relación con la marca; lealtad expresada principalmente en términos de comportamiento revelado; y la compra moderada por las características de los individuos, circunstancias y/o la situación de compra.

La fidelidad o lealtad de un cliente está basada no solo en un comportamiento recurrente y constante, sino también en comportamientos psicológicos que lo atan a determinado producto o servicio, y una de las maneras más asertivas de llegar a un cliente es a través de un trato personalizado, cálido y ágil, lo que es posible gracias al factor trabajo que se encuentra inmerso en todo el proceso.

Determinar la influencia de ciertas variables, en la probabilidad de que un socio sea fiel a una COAC de carácter institucional, es el problema que se estudia en esta investigación, debido a que uno de los principales retos que tienen las COAC es la competencia agresiva que existe por parte de instituciones financieras más grandes, como los bancos u otras COAC. Si bien, con todas las regulaciones impuestas por los entes de control, hasta cierto punto se ha logrado reducir la competencia desleal por tasas de interés, la liquidación de cooperativas ha generado un temor latente en el sector, por lo que se hace imprescindible la medición de la retención y deserción de clientes a través de la fidelización de los asociados. En el caso de las COAC de naturaleza abierta, es más sencillo sobrellevar el efecto de la salida de un socio, pero en las cooperativas de carácter institucional este problema se agranda, puesto que la posibilidad de captar nuevos asociados es limitada, y el impacto en cuanto a retiro de depósitos y reducción de cartera es significativo.

Además del capítulo introductorio, el presente trabajo está conformado por el marco teórico, sección que desarrolla aspectos relevantes sobre la medición de la fidelidad de los clientes desde distintas perspectivas; además, se explica el modelo empleado, con sus respectivas variables y la razón por la cual se emplearon; adicionalmente, en esta misma sección se encontrarán características de la base de datos empleada y de la institución a la cual se aplicará el modelo. A continuación se presentan los principales resultados, con la correspondiente interpretación; y por último, se incorporó una sección de conclusiones, en la que se detallan de manera resumida los aspectos más relevantes que se pudo observar y obtener a lo largo de la investigación. 


\section{Revisión de la literatura}

Uno de los trabajos más relevantes sobre la fidelización de clientes es "Un modelo de la lealtad del cliente en el mercado de la banca minorista", de Beerli, Martín y Quintana (2004), cuyos resultados fueron significativos. Dicho trabajo se centra en la banca minorista como área de estudio, y realiza un comparativo entre la lealtad a la marca y la facilidad de cambio en caso de reducirse los costos de cambio, para lo cual se empleó un modelo de ecuaciones estructurales. Su trabajo partió de la premisa de que la lealtad no es un comportamiento aislado a la psicología humana, además de que la misma no se mide o está representada únicamente por la acción de una compra repetida; para esto se hizo distinción entre dos tipos de conceptualizaciones acerca de la lealtad: por un lado se encuentra aquella fidelización basada en la inercia, es decir, compras algo porque está de moda o porque estás acostumbrado; y por otro lado, se encuentra la lealtad verdadera, también considerada por los autores como lealtad de marca, que consiste en esa característica ligada al comportamiento psicológico de una persona para regresar al mismo lugar y comprar el mismo producto, generando igual o mayor satisfacción que la primera vez que lo hizo. Los autores también hacen un análisis de la influencia de ciertos factores en la lealtad del cliente y no únicamente en la satisfacción, término que generalmente está asociado a la lealtad.

Teniendo en cuenta la gran controversia que existe acerca de si la calidad percibida es un antecedente que ejerce una indirecta influencia en la lealtad y una influencia directa en la satisfacción, o si, por el contrario, es una consecuencia de la satisfacción, hemos tratado de explicar la dirección causal de la relación existente entre la calidad y la satisfacción percibida. Los resultados del modelo propuesto, cuya validez ha sido demostrada por los niveles relativamente aceptables de los indicadores y por su alto valor explicativo, demuestran que tanto la satisfacción y costes de cambio se pueden considerar como antecedentes de fidelidad. Sin embargo, la influencia ejercida por la satisfacción es mucho mayor que la de los costes de cambio. (Beerli, Martín y Quintana 2004, 268)

Otro estudio que aportó de manera relevante a la importancia de la fidelización y de sus variables relacionadas fue "Determinantes de la rotación de suscriptores y la lealtad del cliente en el mercado coreano de telefonía móvil”, de Hee-Su Kim y Choong-Han Yoon (2004), quienes a partir de un 
modelo binomial logit, establecieron variables dicotómicas que permiten establecer los determinantes para que un socio sea fiel a una empresa en particular, hallando como resultados:

A diferencia del modelo de la rotación, la duración de suscripción no tiene un efecto significativo en estos clientes fieles, lo que indica que los suscriptores desde hace mucho tiempo se quedan con sus empresas actuales por pura satisfacción con el desempeño del servicio, y no por el lock-in. Este resultado implica que el efecto lock-in causado por una duración larga de suscripción se concentra en aquellos clientes que desean permanecer con su actual compañía, pero no son lo suficientemente leales a la empresa. El efecto lock in para este grupo de clientes probablemente proviene de los costes de cambio. En el modelo de rotación, el resultado de la estimación de un coeficiente positivo para la duración de teléfono utilizado indica que cuanto más tiempo un suscriptor ha utilizado el presente auricular, es más probable que cambien de proveedor. (Kim y Yoon 2004, 764)

Guadagni y Little (1983), en su texto "A Logit Model of Brand Choice Calibrated on Scanner Data", buscaron observar la lealtad de los clientes a su marca de café utilizando un modelo logit:

El modelo logit multinominal ha proporcionado una excelente representación del mercado regular de café molido a nivel de clientes individuales y tiendas minoristas. Los coeficientes del modelo son estadísticamente significativos, más altos. El modelo calibrado predice el comportamiento de una muestra de clientes en espera. Una característica destacable del modelo es su parsimonia. Los principales coeficientes son la lealtad de marca y el tamaño, y el control de las variables son las mismas para todos los tamaños de marca y clientes, y sin embargo el modelo predice hábilmente los tamaños de marca con acciones ampliamente diferentes, sigue diferentes tendencias y cambios en el tiempo y rastrea el rendimiento de la marca en diferentes tipos de víveres. Los resultados no son perfectos, ya que varibles de marketing no son consideradas (por ejemplo, calidad de visualización, cupones y medios), como sabemos por otros estudios que estas acciones influyen en la decisión de compra. Sin embargo, los resultados aquí parecen muy prometedores. (Guadagni y Little 1983, 233)

En esta área, es justo hacer mención a otros estudios como Guadarrama y Rosales (2015), Osses (2015), Peña, Ramírez y Osorio (2014), y Zins (2001), quienes, si bien no encontraron resultados significativos en el estudio de la fidelización de clientes, sirvieron como base para fortalecer el modelo de esta investigación, desechando aquellas variables poco significativas. 
La fidelización permite a una empresa entender qué clientes son una base segura y cuáles son solo pasajeros, lo que ayuda a crear escenarios distintos tanto para mantener esa fidelidad como para lograr integrar nuevos socios a esa calidad.

Cuando hablamos de fortalecer la relación con los clientes muchas veces se pone el acento en aspectos técnicos, en cuestiones comerciales, en lograr que el cliente repita ventas con nosotros ofreciendo descuentos, redes sociales, etc. Pero pocas veces se valora el factor humano para fidelizar al cliente. [...] no solo hay que conocer al cliente, sino que además hay que ser lo suficientemente cercanos para que ellos se sientan cómodos con nosotros. Se establece una relación comercial, no se trata de ser amigos, pero sí de estar al lado del cliente y empatizar con lo que nos pide, ofreciendo la mejor solución viable en ese momento. (Roberto 2018, párr. 2)

La fidelización en las COAC de carácter institucional en Ecuador no solo se encuentra ligada a la satisfacción que le genera un determinado producto o servicio; por el contrario, existe un comportamiento muy particular de los socios y el apego o satisfacción a la institución asociado con el trato que reciben por parte del personal a cargo; personal que es el encargado de generar el ambiente de familiaridad que causa un gran efecto al momento de la decisión de fidelidad de un socio.

\section{Metodología}

La base de datos empleada en la presente investigación consiste en información proporcionada por la Cooperativa de Ahorro y Crédito Ciudad de Quito, una entidad que lleva en el mercado 56 años de trayectoria y es de carácter institucional, siendo sus socios empleados de instituciones relacionadas con el Gobierno de los Estados Unidos de América, tales como Embajada Americana, el Colegio Americano, la Academia Cotopaxi, Conservación Internacional, Cuerpo de Paz y Colegio Saint Patrick. Es importante destacar que los empleados que se jubilan o terminan su relación contractual con las instituciones pueden continuar siendo parte de las COAC, por la que hay un gran porcentaje de socios varios. En total existen 465 observaciones correspondientes a todos los aso ciados que, para la fecha de corte de diciembre de 2018, eran parte de la COAC. 
En el modelo se aplicaron las siguientes variables dummy: sexo, siendo 1 femenino y 0 masculino; e institución, donde el valor de 1 indica que es parte de alguna institución vinculada con el Gobierno de los Estados Unidos. Además, se emplearon seis variables discretas que contemplan características particulares de los asociados, tales como edad; cuentas, que engloba el número de cuentas de ahorros que el asociado mantiene con la institución; créditos, que describe el número de créditos vigentes que el asociado tiene en la COAC a la fecha de corte; antigüedad, que permite identificar cuántos años llevan siendo parte de la cooperativa, y se la obtuvo restando la fecha de ingreso menos el año de corte (2018); la variable $m$ _ahorros contiene el monto total de ahorros que mantiene cada uno de los asociados en sus distintas cuentas dentro de la institución; y $m$ _creditos contiene el saldo total de crédito que tiene el asociado en la institución.

También se emplearon dos variables con escalas: instrucción, donde educación primaria es 1 , secundaria es 2 , tercer nivel es 3 , maestría es 4 ; para la variable estado_civil, la escala empleada es: soltero $=1$, casado $=2$, viudo $=$ 3 , unión libre $=4$ y divorciado $=5$.

La variable dependiente se ha denominado lealtad y es una variable dicotómica que contempla valores de 0 y 1 ; en el caso de que sea 0 quiere decir que su probabilidad de lealtad es mínima; y en el caso de que sea 1 es que existe una probabilidad significativa de que el socio sea leal. Para determinar en la base de datos si un socio es leal o no, se emplearon tres criterios propios de la institución:

1. Antigüedad del asociado, socios que no mantienen cuentas inactivas, es decir, todas las cuentas tienen movimiento de manera frecuente.

2. Monto de dinero que mantiene en ahorros, situando como valor de mayor probabilidad de ser leal aquellos ahorros que sean igual o mayor a USD 20.000; es importante destacar que la institución no maneja depósitos a plazo fijo.

3. Monto de créditos que mantiene el socio en la institución, con un monto de USD 20.500 en adelante.

Para estimar los parámetros de este modelo se empleó un modelo logit, el mismo que permitió evaluar la influencia de determinadas variables en la probabilidad de que un socio sea fiel o no a la COAC. El modelo logit tiene 
como característica que emplea una distribución logística y que la variable dependiente es dicotómica.

El modelo "Logit" es una herramienta de estimación que surge ante la necesidad de evaluar la probabilidad de que un individuo adopte un parámetro establecido $(\mathrm{Y}=1)$ de acuerdo con los comportamientos de las variables independientes. Para lo cual el modelo considera una distribución logística en donde la variable explicada es dicótoma tomando valores en el rango entre 0 y 1 y las variables independientes pueden ser diversas de acuerdo al objetivo de análisis. (Novales 2010,540)

El modelo empleado fue el siguiente:

logit lealtad $=\beta o+\beta 1$ edad $+\beta 2$ sexo $+\beta 3$ cuentas $+\beta 4$ creditos $+\beta 5$ antiguedad

$+\beta 6$ instruccion $+\beta 7$ institucion $+\beta 8$ estado_civil $+\beta 9 m_{-}$ahorros $+\beta 10 m_{-}$creditos

\section{El software empleado fue Stata.}

Se aplicó una prueba de robustez para probar la validez del modelo antes de interpretar sus resultados. Para este fin se empleó la curva Receiver Operating Characteristic (ROC), caracterizada como "la curva de características operativas del receptor (ROC), que se define como un gráfico de la sensibilidad como la coordenada, y frente a su especificidad 1 o tasa de falsos positivos (FPR) como la coordenada $\mathrm{x}$, es un método eficaz para evaluar el rendimiento de pruebas diagnósticas" (Park, Goo y Jo 2004, 12).

La matriz de confusión es un método muy empleado para clasificar a un modelo, el mismo que tiene como objetivo identificar si las predicciones realizadas en un modelo fueron acertadas y cuántas no lo fueron, para lo cual es necesario una comparación entre lo pronosticado y lo real.

Kohavi \& Provost definieron la matriz de confusión como un conjunto de información de clasificación actual. En donde la matriz de confusión contiene información actual y predicciones realizada por un sistema de clasificación. El rendimiento de este sistema es evaluado usando información contenida en la matriz. Los datos de la matriz de clasificación tienen los siguientes significados: a) es el número de predicciones correctas en la cual un dato es negativo; b) es el número de predicciones incorrectas en la cual un dato es positivo; c) es el número de predicciones incorrectas en la cual un dato es negativo; d) es el número de predicciones correctas en la cual un dato es positivo. (Restrepo y Orozco 2015, 53) 
Se aplicaron también las pruebas que permiten identificar la existencia de multicolinealidad, heteroscedasticidad y existencia de normalidad en los errores.

\section{Resultados}

Una vez que se aplicó la regresión, los resultados obtenidos fueron los siguientes:

Tabla 1

Resultados del modelo logit de fidelización

\begin{tabular}{|c|c|c|c|c|c|}
\hline \multicolumn{4}{|c|}{ Número de observaciones } & \multicolumn{2}{|r|}{465} \\
\hline \multicolumn{4}{|l|}{ LR chi2(10) } & \multicolumn{2}{|c|}{473,67} \\
\hline \multicolumn{4}{|l|}{ Prob> chi2 } & \multicolumn{2}{|c|}{0,0000} \\
\hline \multicolumn{4}{|l|}{ Pseudo R2 } & & 7757 \\
\hline Coeficiente & Error estándar & $z$ & $p>|z|$ & \multicolumn{2}{|c|}{ (95\% Intervalo de confianza) } \\
\hline$-0,0249428$ & 0,292017 & $-0,85$ & 0,393 & $-0,82177$ & 0,0322914 \\
\hline 0,765592 & 0,4734568 & 1,62 & 0,106 & $-0,1623662$ & 1,69355 \\
\hline$-0,2447724$ & 0,4082612 & $-0,60$ & 0,549 & $-1,04495$ & 0,5554049 \\
\hline$-1,17714$ & 0,3979451 & $-2,96$ & 0,003 & $-1,957099$ & $-0,3971823$ \\
\hline 0,3973334 & 0,579678 & 6,85 & 0,000 & 0,2837186 & 0,5109483 \\
\hline 0,153203 & 0,4510307 & 0,03 & 0,973 & $-0,8686837$ & 0,899243 \\
\hline$-0,7599343$ & 0,6660993 & $-1,14$ & 0,254 & $-2,065465$ & 0,5455964 \\
\hline 0,1495463 & 0,1799314 & 0,83 & 0,406 & $-0,2031127$ & 0,5022053 \\
\hline 0,0001403 & 0,000264 & 5,32 & 0,000 & 0,0000886 & 0,000192 \\
\hline 0,0003206 & 0,0000542 & 5,92 & 0,000 & 0,0002144 & 0,0004268 \\
\hline$-7,451351$ & 2,446604 & $-3,05$ & 0,002 & $-12,24661$ & $-2,656095$ \\
\hline
\end{tabular}

Elaboración propia.

A partir de estos resultados se puede observar que, en conjunto, el modelo es significativo puesto que la prob $>$ chi2 es menor a 0,05 . Posteriormente, se obtuvieron los efectos marginales de la regresión. 
Tabla 2

Efectos marginales después de aplicar logit

\begin{tabular}{|l|c|c|c|c|r|r|r|}
\hline \multicolumn{1}{|c|}{ Variable } & dy/dx & $\begin{array}{c}\text { Error } \\
\text { estándar }\end{array}$ & $\mathbf{Z}$ & $\mathbf{p}>|\mathbf{z}|$ & \multicolumn{2}{c|}{$\begin{array}{c}\text { (95\% Intervalo } \\
\text { de confianza) }\end{array}$} & $\mathbf{X}$ \\
\hline Edad & $-0,0047573$ & 0,0055 & $-0,86$ & 0,387 & $-0,01554$ & 0,006025 & 48,1441 \\
\hline Sexo* & 0,1385365 & 0,08313 & 1,67 & 0,096 & $-0,024393$ & 0,301466 & 0,630108 \\
\hline Cuentas & $-0,0466848$ & 0,0783 & $-0,60$ & 0,551 & $-0,200152$ & 0,106782 & 1,81935 \\
\hline Créditos & $-0,2245129$ & 0,07753 & $-2,90$ & 0,004 & $-0,376475$ & $-0,07255$ & 0,845161 \\
\hline Antigüedad & 0,0757823 & 0,1177 & 6,44 & 0,000 & 0,052711 & 0,098854 & 11,8065 \\
\hline Instrucción & 0,002922 & 0,08616 & 0,03 & 0,973 & $-0,165956$ & 0,1718 & 2,56559 \\
\hline Institución* & $-0,1613478$ & 0,15297 & $-1,05$ & 0,292 & $-0,461165$ & 0,138469 & 0,845161 \\
\hline Estado_civil & 0,0285226 & 0,03358 & 0,85 & 0,396 & $-0,037295$ & 0,09434 & 2,14839 \\
\hline M_ahorros & 0,0000268 & 0,00001 & 4,67 & 0,000 & 0,000016 & 0,000038 & 10517,20 \\
\hline M_créditos & 0,000611 & 0,00001 & 5,61 & 0,000 & 0,00004 & 0,000083 & 8300,69 \\
\hline *dy/dx es para el cambio de variables discretas a variables dummy de 0 a 1. \\
\hline
\end{tabular}

Elaboración propia.

Las variables significativas del modelo fueron el número de créditos, la antigüedad de los socios en la institución, el monto total de los ahorros y el monto total de los créditos. Esto se puede determinar debido a que la probabilidad del valor $\mathrm{z}$ en las cuatro variables es menor al $5 \%$, es decir, adoptan valores que son menores a 0,05 .

Si se interpretan los coeficientes de las variables empleadas, se puede determinar que un año más de edad provoca que la probabilidad de lealtad o retención de un socio disminuya en $0,4 \%$, es decir, mientras un socio tiene mayor edad, su nivel de fidelidad empieza a disminuir; en el caso del sexo, los hombres tienen mayor probabilidad de fidelizarse con la cooperativa en un $13,85 \%$, en el caso de una mujer, la probabilidad disminuye en igual proporción.

Por cada cuenta adicional que apertura un asociado en la cooperativa, su fidelidad disminuirá en un $4,66 \%$; por cada crédito adicional que solicite un asociado, su fidelidad disminuye en un $22,45 \%$. Esto puede darse porque el 
socio necesita dinero, y al tener cupos máximos en la institución puede ampliar su historial crediticio en otras instituciones; un año más de antigüedad de un asociado con la cooperativa aumenta en un 7,58\% la probabilidad de fidelidad, puesto que entre más años tiene un asociado en la institución mayor es su apego y fidelidad.

Un año más de escolaridad en los socios incrementa su probabilidad de fidelización en un $0,0292 \%$. Esto puede darse porque se tendría mayor información de su cooperativa. Los socios categorizados como varios tienen una probabilidad de $1,61 \%$ de ser más fieles o leales con la cooperativa, caso contrario sucede con los socios que trabajan en otra institución, para quienes disminuye la probabilidad de ser fieles en la misma proporción. Esto puede darse debido a que si un socio decide mantenerse en la cooperativa a pesar de haber abandonado el vínculo que generó su ingreso es porque es más leal con la misma. Por cada dólar adicional al monto de ahorros de un socio, la probabilidad de fidelidad se incrementa en un $0,00268 \%$, mientras que si el monto del crédito de un socio incrementa en un dólar, la probabilidad de fidelidad del mismo incrementa en un $0,00611 \%$.

Para medir la sensibilidad en el modelo es preciso aplicar la curva ROC; por un lado, se encuentran las pruebas de igualdad de áreas ROC, y por otro lado, las pruebas de igualdad de áreas ROC contra una curva ROC estándar.

Figura 1

\section{Curva ROC}

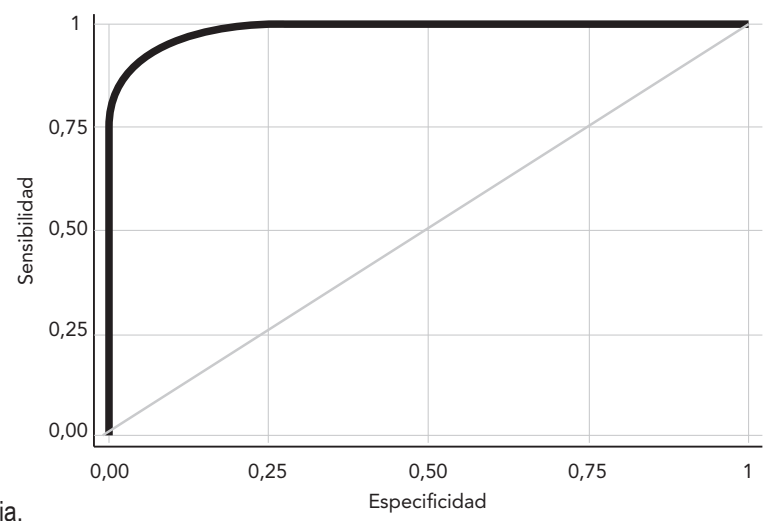

Elaboración propia. 
A partir de la curva obtenida en la figura 1 (anexo), se puede determinar que, al encontrarse por encima de la diagonal, los resultados obtenidos muestran una cantidad mínima de falsos negativos, es decir, que aquellos puntos obtenidos representan resultados mejores que si se hubiesen generado datos al azar.

Tabla 3

Pruebas de igualdad de áreas ROC

\begin{tabular}{|l|c|c|c|c|c|}
\hline & \multicolumn{3}{|c|}{ ROC } & \multicolumn{1}{c|}{} \\
\cline { 2 - 6 } & Obs & Área & Error estándar & (95\% Conf. Interval) \\
\hline Edad & 465 & 0,7624 & 0,0233 & 0,71669 & 0,80810 \\
\hline Sexo & 465 & 0,5365 & 0,0230 & 0,49154 & 0,58154 \\
\hline Cuentas & 465 & 0,6316 & 0,0207 & 0,59099 & 0,67216 \\
\hline Créditos & 465 & 0,5628 & 0,0264 & 0,51111 & 0,61445 \\
\hline Antigüedad & 465 & 0,9240 & 0,0118 & 0,90093 & 0,94708 \\
\hline Instrucción & 465 & 0,3024 & 0,0230 & 0,25742 & 0,34745 \\
\hline Institución & 465 & 0,3810 & 0,0192 & 0,34337 & 0,41855 \\
\hline Estado_civil & 465 & 0,6927 & 0,0212 & 0,65103 & 0,73433 \\
\hline M_ahorros & 465 & 0,7868 & 0,0230 & 0,74179 & 0,83185 \\
\hline M_creditos & 465 & 0,6200 & 0,0283 & 0,56452 & 0,67548 \\
\hline Chi2(9) = & \multicolumn{2}{|c|}{1157,43} & Prob>chi2= & \multicolumn{3}{c|}{0,0000} \\
\hline
\end{tabular}

Elaboración propia.

Se observa que las variables con mayor probabilidad de que el diagnóstico sea correcto son antigüedad, monto de ahorros y edad, mientras que las variables cuyo diagnóstico está más cercano al azar son instrucción, institución, sexo y créditos.

Una vez que se han analizado todos los resultados obtenidos, la importancia del modelo es evidenciable, puesto que permitirá a la cooperativa apuntar sus esfuerzos para retener a aquellos socios que tienen mayor probabilidad de lealtad en la cooperativa, estos son los que tienen mayor antigüedad y cuyos montos totales de ahorros y créditos son mayores, demostrando 
que no siempre el número de operaciones es símbolo de lealtad. También se encontró que los hombres tienen mayor probabilidad de lealtad, por lo que la institución puede crear productos o servicios orientados a ese sector.

Es importante entender que el modelo de retención no tiene como objetivo descuidar a aquellos socios que tienen menor probabilidad de lealtad; por el contrario, es necesario trabajar para lograr una inserción de estos grupos.

\section{Conclusiones}

A partir del modelo empleado se determinó que, si bien existieron variables que no se pueden considerar significativas, el modelo en su conjunto sí es significativo al mostrar un patrón claro de que la variable con mayor influencia en la fidelización de los clientes para una cooperativa de ahorro y crédito de carácter institucional es la antigüedad de los socios. Esto, si bien parece un aspecto lógico, no siempre es señal de fidelidad. Por otro lado, variables como el monto total de ahorros y créditos que el socio mantiene en la institución son factores de influencia significativa dentro de la lealtad del socio.

Asociando los resultados obtenidos con la importancia del factor trabajo dentro de la fidelización de los socios a una cooperativa de ahorro y crédito de carácter institucional, se pudo observar que aquellas variables más significativas estadísticamente, dentro del modelo, tienen una relación directa con el capital humano en la institución. Por ejemplo, en el caso del monto de ahorros de un socio, este se encuentra muy ligado a la seguridad que la organización genera $\mathrm{y}$, por consiguiente, al nivel de apego o conocimiento que tiene de la institución. Es ahí donde se integra el componente del factor humano. Las cifras financieras y las tasas de interés no son el único aliciente para que un socio coloque grandes montos de dinero en una cooperativa, dependerá mucho de la persona que tome contacto con el socio y logre convencerlo de colocar estos fondos en la institución, y del equipo de trabajo, a través de los registros contables ágiles, oportunos y eficientes, de tal manera que el socio pueda llevar un control de sus cuentas garantizando seguridad en su dinero, entre otros aspectos que reflejan este tipo de comportamiento. Otra de las variables estadísticamente significativas es el monto de créditos obtenidos. 
En este caso se revela nuevamente la influencia del capital de trabajo, considerando que para que un socio decida hacer crédito en la cooperativa debió tener un acercamiento directo con uno de los funcionarios, en este caso el oficial de crédito, quien no solo dio un seguimiento al crédito, sino que también trasladó todos los beneficios que el crédito podría ofrecer al asociado.

Una de las variables con mayor relevancia dentro del modelo de lealtad fue la antigüedad, es decir, la permanencia en una institución a pesar de la fluctuación de tasas de interés es un indicador de la seguridad en la institución. Todos los ejemplos citados son claras muestras de que si bien el factor trabajo, específicamente del capital humano, no es considerado como generador de ganancias a partir del lado contable, la influencia de las mismas, a través de la lealtad y fidelidad de los asociados, es real. La fidelización de clientes es un tema de gran relevancia en el sector financiero, como en otros sectores de la economía cuyo financiamiento depende de los clientes.

El modelo presentado tuvo como objetivo mostrar la fidelidad de clientes desde una perspectiva estadística, pero en futuras investigaciones este podría servir de base para un modelo de consistencia financiera, para lo cual se deberían cambiar el tipo de modelo empleado e incorporar otro tipo de variables.

\section{Referencias}

Beerli, Asunción, Josefa Martin y Agustín Quintana. 2004. “A Model of Customer Loyalty in the Retail Banking Market”. European Journal of Marketing 38 (2): 253-275. Doi: 10.1108/03090560410511221.

EC Ministerio de Inclusión Económica y Social (MIES). 2013. "El 60\% de empleo en el Ecuador lo genera la economía popular y solidaria”. Ministerio de Inclusión Económica y Social. Consulta: mayo de 2019. 〈https://bit.ly/2OWydBV〉.

EC Superintendencia de Economía Popular y Solidaria (SEPS). 2014. "Serie Estudios sobre economía popular y solidaria: contextos de la otra economía". Superintendencia de Economía Popular y Solidaria. Consulta: mayo de 2019. 〈https://bit.ly/2ARFVoj〉.

EC. 2014. Código Orgánico Monetario y Financiero. Registro Oficial 298, Segundo Suplemento, 12 de octubre.

Guadagni, Peter, y John Little. 2008. “A Logit Model of Brand Choice Calibrated on Scanner Data”. Marketing Science 27 (1): 29-48. Doi: 10.1287/mksc.1070.0331. 
Guadarrama, Enrique. 2015. "Propuesta de un modelo de valoración del cliente desde la perspectiva marketing-rentabilidad para aplicar en empresas turísticas". RICIT: Revista Turismo, Desarrollo y Buen vivir 9 (2): 38-59. «https://dialnet.unirioja.es/servlet/articulo? codigo $=5309453$ >.

Guadarrama, Enrique, y Mireya Rosales. 2015. "Marketing relacional: valor, satisfacción, lealtad y retención del cliente. Análisis y reflexión teórica”. Ciencia y Sociedad 40 (2): 307-340. 〈https://www.redalyc.org/articulo.oa?id=87041161004〉.

Kim, Hee Su, y Yoon Choong Han. 2004. "Determinants of Subscriber Churn and Customer Loyalty in the Korean Mobile Telephony Market". Telecommunications Policy 28 (9): 751-765. Doi: 10.1016/j.telpol.2004.05.013.

Novales, Alfonso. 2010. Econometría. Madrid: McGraw-Hill.

Osses Godoy, Alex Alfredo. 2015. "Desarrollo de un método de valoración de clientes en una empresa del sector automotriz". Tesis de pregrado, Universidad de Chile, Santiago de Chile. 〈https://bit.ly/2MuVwjo〉.

Park, Seong Ho, Jin Mo Goo y Chan-Hee Jo. 2004. "Receiver Operating Characteristic (ROC) Curve: Practical Review for Radiologists”. Korean J Radiol 5 (1): 11-18. Doi:10.3348/ kjr.2004.5.1.11.

Peña, Stefanía, Gloria Ramírez y Juan Osorio. 2015. "Evaluación de una estrategia de fidelización de clientes con dinámica de sistemas". Revista Ingenierías Universidad de Medellin 14 (26): 87-104. Doi: 〈https://doi.org/10.22395/rium.v14n26a6〉.

Restrepo, Andrés, y Santiago Orozco. 2015. "Modelo predictivo de quiebra para bancos estadounidenses". Tesis posgrado, Colegio de Estudios Superiores de Administración, Sede Bogotá. 〈https://bit.ly/32bhJcG〉.

Roberto, Carlos (Pymes y Autónomos). 2018. "El factor humano en la relación con el cliente, clave en la fidelización". PymesyAutónomos. Consulta: abril de 2019. «https://bit.ly/2Auhay9>.

Uncles, Mark, Grahame Dowling y Kathy Hammond. 2003. "Customer Loyalty and Customer Loyalty Programs". Journal of Consumer Marketing 20 (4): 294-319. Doi: 10.1108/07363760310483676.

Zins, Andreas. 2001. "Relative Attitudes and Commitment in Customer Loyalty Models". International Journal of Service Industry Management 12 (3): 269-294. Doi: 10.1108/ eum0000000005521. 


\section{Anexo \\ Tabla 1 \\ Identificación de las variables en el modelo}

\begin{tabular}{|c|c|c|c|c|c|}
\hline Variable & Observación & Media & Desviación estándar & Min. & Max. \\
\hline Lealtad & 465 & 0,3655914 & 0,4821143 & 0 & 1 \\
\hline Edad & 465 & 48,14409 & 13,24157 & 19 & 94 \\
\hline Sexo & 465 & 0,6301075 & 0,4832953 & 0 & 1 \\
\hline Cuentas & 465 & 1,819355 & 0,6644057 & 0 & 6 \\
\hline Créditos & 465 & 0,8451613 & 0,9922671 & 0 & 5 \\
\hline Antigüedad & 465 & 11,80645 & 12,34565 & 0 & 55 \\
\hline Instrucción & 465 & 2,565591 & 0,5614203 & 1 & 4 \\
\hline Institución & 465 & 0,8451613 & 0,3621405 & 0 & 1 \\
\hline Estado_civil & 465 & 2,148387 & 1,202334 & 1 & 5 \\
\hline M_ahorros & 465 & 10517,22 & 15743,72 & 0.09 & 113571,50 \\
\hline M_créditos & 465 & 8300,687 & 20521,46 & 0 & 387385 \\
\hline
\end{tabular}

Elaboración propia.

Tabla 2

\section{Curva ROC}

\begin{tabular}{|c|c|c|c|c|c|}
\hline \multicolumn{6}{|c|}{ Pruebas de igualdad de áreas ROC } \\
\hline \multicolumn{6}{|c|}{$\begin{array}{l}\text { Roccomp: lealtad, edad, sexo, cuentas, créditos, antigüedad, instrucción, institución, esta- } \\
\text { do_civil, m_ahorros, m_créditos. }\end{array}$} \\
\hline & \multicolumn{3}{|c|}{ ROC } & & \\
\hline & Observación & Área & Error estándar & \multicolumn{2}{|c|}{ (95\% Intervalo de confianza) } \\
\hline Edad & 465 & 0,7624 & 0,0233 & 0,71669 & 0,80810 \\
\hline Sexo & 465 & 0,5365 & 0,0230 & 0,49154 & 0,58154 \\
\hline Cuentas & 465 & 0,6316 & 0,0207 & 0,59099 & 0,67216 \\
\hline Créditos & 465 & 0,5628 & 0,0264 & 0,51111 & 0,61445 \\
\hline
\end{tabular}


Fidelización en el sector cooperativo de carácter institucional en Ecuador

\begin{tabular}{|l|l|l|l|l|l|}
\hline Antigüedad & 465 & 0,9240 & 0,0118 & 0,90093 & 0,94708 \\
\hline Instrucción & 465 & 0,3024 & 0,0230 & 0,25742 & 0,34745 \\
\hline Institución & 465 & 0,3810 & 0,0192 & 0,34337 & 0,41855 \\
\hline Estado_civil & 465 & 0,6927 & 0,0212 & 0,65103 & 0,73433 \\
\hline M_ahorros & 465 & 0,7868 & 0,0230 & 0,74179 & 0,83185 \\
\hline M_créditos & 465 & 0,6200 & 0,0283 & 0,56452 & 0,67548 \\
\hline \multicolumn{1}{|c|}{ Chi2(9)= } & \multicolumn{2}{|c|}{1157.43} & Prob>chi2 $=$ & \multicolumn{2}{c|}{0,0000} \\
\hline
\end{tabular}

Elaboración propia.

Tabla 3

\section{Pruebas de igualdad de áreas ROC contra una curva ROC estándar}

Rocgold: lealtad, edad, sexo, cuentas, créditos, antigüedad, instrucción, institución, estado_civil, m_ahorros, m_créditos.

\begin{tabular}{|l|c|c|r|c|c|c|}
\hline & ROC & & & & & Bonferroni \\
\hline & Área & Error estándar & \multicolumn{1}{c|}{ Chi2 } & df & Pr>chi2 & Pr>xhi2 \\
\hline Edad (estándar) & 0,7624 & 0,0233 & & & & \\
\hline Sexo & 0,5365 & 0,0230 & 52,4160 & 1 & 0,0000 & 0,0000 \\
\hline Cuentas & 0,6316 & 0,0207 & 20,3269 & 1 & 0,0000 & 0,0001 \\
\hline Créditos & 0,5628 & 0,0264 & 25,0870 & 1 & 0,0000 & 0,0000 \\
\hline Antigüedad & 0,9240 & 0,0118 & 74,7850 & 1 & 0,0000 & 0,0000 \\
\hline Instrucción & 0,3024 & 0,0230 & 158,0894 & 1 & 0,0000 & 0,0000 \\
\hline Institución & 0,3810 & 0,0192 & 128,6625 & 1 & 0,0000 & 0,0000 \\
\hline Estado_civil & 0,6927 & 0,0212 & 6,3647 & 1 & 0,0116 & 0,1048 \\
\hline M_ahorros & 0,7868 & 0,0230 & 0,6278 & 1 & 0,4282 & 1,0000 \\
\hline M_créditos & 0,6200 & 0,0283 & 11,2090 & 1 & 0,0008 & 0,0073 \\
\hline
\end{tabular}

Elaboración propia. 
Tabla 4

Matriz de confusión

\begin{tabular}{|c|c|c|}
\hline \multicolumn{3}{|l|}{ *Matriz de confusión } \\
\hline \multicolumn{3}{|l|}{ estat classification } \\
\hline \multicolumn{3}{|l|}{ Logistic model for lealtad } \\
\hline & -TRUE- & \\
\hline Classified & $\sim D$ & Total \\
\hline 154 & 11 & 165 \\
\hline 16 & 284 & 300 \\
\hline Total & 295 & 465 \\
\hline \multicolumn{3}{|c|}{ Classified + if predicted $\operatorname{Pr}(D)>=0,5$} \\
\hline \multicolumn{3}{|l|}{ True $D$ defined as lealtad $!=0$} \\
\hline Sensitivity & $\operatorname{Pr}(+\mid D)$ & $90,59 \%$ \\
\hline Specificity & $\operatorname{Pr}(-\mid \sim D)$ & $96,27 \%$ \\
\hline Positive predictive value & $\operatorname{Pr}(\mathrm{D} \mid+)$ & $93,33 \%$ \\
\hline Negative predictive value & $\operatorname{Pr}(\sim \mathrm{D} \mid-)$ & $94,67 \%$ \\
\hline False + rate for true $\sim D$ & $\operatorname{Pr}(+\mid \sim D)$ & $3,73 \%$ \\
\hline False - rate for true $D$ & $\operatorname{Pr}(-\mid \mathrm{D})$ & $9,41 \%$ \\
\hline False + rate for classified + & $\operatorname{Pr}(\sim \mathrm{D} \mid+)$ & $6,67 \%$ \\
\hline False - rate for classified - & $\operatorname{Pr}(\mathrm{D} \mid-)$ & $5,33 \%$ \\
\hline \multicolumn{2}{|l|}{ Correctly classified } & $94,19 \%$ \\
\hline
\end{tabular}

Elaboración propia. 
Figura 1

Curva de sensibilidad y especificidad

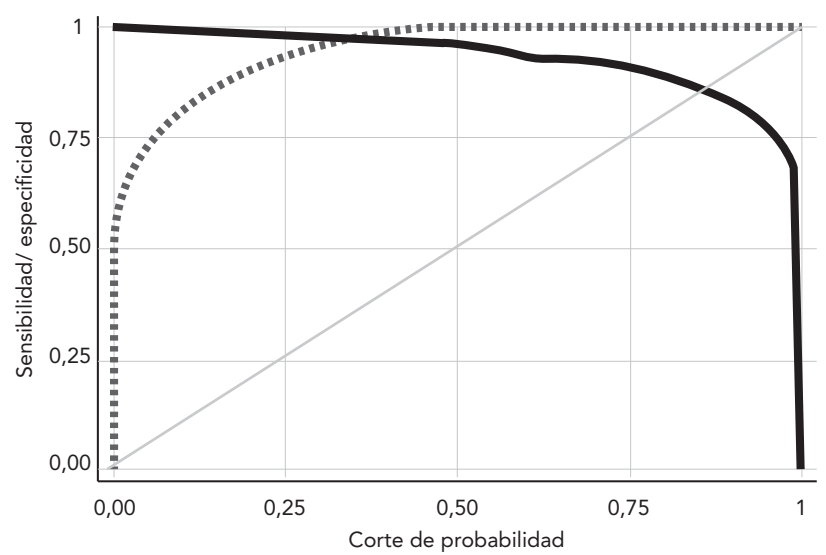

\begin{tabular}{|l|l|}
\hline Sensibilidad & Especificidad Munumu \\
\hline
\end{tabular}

Tabla 5

Multicolinealidad

\begin{tabular}{|l|c|c|}
\hline \multicolumn{3}{|c|}{ Vif } \\
\hline \multicolumn{1}{|c|}{ Variable } & VIF & 1/VIF \\
\hline Antigüedad & 2,93 & 0,341793 \\
\hline Edad & 2,06 & 0,484755 \\
\hline Institución & 1,42 & 0,704863 \\
\hline Instrucción & 1,31 & 0,762133 \\
\hline Créditos & 1,30 & 0,770837 \\
\hline M_créditos & 1,28 & 0,783268 \\
\hline M_ahorros & 1,24 & 0,804870 \\
\hline Estado_civil & 1,23 & 0,814160 \\
\hline Cuentas & 1,19 & 0,841851 \\
\hline Sexo & 1,02 & 0,976106 \\
\hline \multicolumn{2}{|c|}{ Mean VIF } & 1,50 \\
\hline
\end{tabular}

Elaboración propia. 


\section{Tabla 6}

\section{Correlación}

\begin{tabular}{|c|c|c|c|c|c|c|c|}
\hline \multicolumn{8}{|c|}{$\begin{array}{l}\text { Corr lealtad, edad, sexo, cuentas, créditos, antigüedad, instrucción, institución, estado_civil, m_ahorros, } \\
\text { m_créditos. }\end{array}$} \\
\hline \multicolumn{8}{|l|}{ (obs=465) } \\
\hline & Lealtad & Edad & Sexo & Cuentas & Créditos & Antigüedad & Instrucción \\
\hline Lealtad & 1,0000 & & & & & & \\
\hline Edad & 0,4451 & 1,0000 & & & & & \\
\hline Sexo & 0,0729 & 0,0582 & 1,0000 & & & & \\
\hline Cuentas & 0,2537 & 0,2217 & 0,0868 & 1,0000 & & & \\
\hline Créditos & 0,1366 & $-0,1005$ & $-0,0792$ & 0,1438 & 1,0000 & & \\
\hline Antigüedad & 0,7039 & 0,7047 & 0,0769 & 0,3213 & $-0,0332$ & 1,0000 & \\
\hline Instrucción & $-0,3515$ & $-0,3763$ & $-0,0454$ & $-0,1935$ & $-0,0398$ & $-0,4465$ & 1,0000 \\
\hline Institución & $-0,3170$ & $-0,4030$ & $-0,0570$ & $-0,0896$ & 0,0291 & $-0,5312$ & 0,2197 \\
\hline Estado_civil & 0,3152 & 0,3011 & 0,0205 & 0,1523 & 0,0681 & 0,3977 & $-0,2779$ \\
\hline M_ahorros & 0,4953 & 0,2403 & 0,0419 & 0,2393 & $-0,0205$ & 0,4021 & $-0,2702$ \\
\hline \multirow[t]{2}{*}{ M_créditos } & 0,2317 & $-0,0754$ & $-0,0918$ & 0,1073 & 0,4460 & 0,0010 & $-0,0851$ \\
\hline & Institución & $\begin{array}{c}\text { Estado } \\
\text { civil }\end{array}$ & $\begin{array}{c}M \\
\text { ahorros }\end{array}$ & $\begin{array}{c}M_{-} \\
\text {créditos }\end{array}$ & & & \\
\hline Institución & 1,0000 & & & & & & \\
\hline Estado_civil & $-0,2540$ & 1,0000 & & & & & \\
\hline M_ahorros & $-0,1839$ & 0,2212 & 1,0000 & & & & \\
\hline M_créditos & 0,0301 & 0,0006 & 0,0366 & 1,0000 & & & \\
\hline
\end{tabular}

Elaboración propia.

Tabla 7

\section{Heteroscedasticidad}

\begin{tabular}{|l|}
\hline Breusch-Pagan / Cook-Weisberg test for heteroskedasticity \\
\hline Ho: Constant variance \\
\hline Variables: fitted values of lealtad \\
\hline Chi2(1) $=35,67$ \\
\hline
\end{tabular}




\begin{tabular}{|c|c|c|c|}
\hline \multicolumn{4}{|l|}{ Prob >chi2 $=0,0000$} \\
\hline \multicolumn{4}{|l|}{ **Test White } \\
\hline \multicolumn{4}{|l|}{ Imtest, white } \\
\hline \multicolumn{4}{|c|}{ White's test for Ho: homoscedasticity } \\
\hline \multicolumn{4}{|c|}{ Against Ha: unrestricted heteroscedasticity } \\
\hline \multicolumn{4}{|l|}{ Chi2 (63) $=264,89$} \\
\hline \multicolumn{4}{|l|}{ Prob> chi2 $=0,0000$} \\
\hline \multicolumn{4}{|c|}{ Cameron \& Trivedi's decomposition of IM-test } \\
\hline Source & Chi2 & $d f$ & $P$ \\
\hline Heteroskedasticity & 264,89 & 63 & 0,0000 \\
\hline Skewness & 159,39 & 10 & 0,0000 \\
\hline Kurtosis & 1,17 & 1 & 0,2784 \\
\hline Total & 425,45 & 74 & 0,0000 \\
\hline
\end{tabular}

Elaboración propia.

Tabla 8

\section{Normalidad de los errores}

\begin{tabular}{|l|c|c|c|c|c|}
\hline \multicolumn{7}{|c|}{ Skewness/Kurtosis tests for Normality } \\
\hline Variable & Obs & $\operatorname{Pr}($ Skewness) & $\operatorname{Pr}$ (Kurtosis) & Adj chi2 (2) & Prob>chi2 \\
\hline R1 & 465 & 0,0000 & 0,2405 & 16,33 & 0,0003 \\
\hline **Prueba Shapiro-Wilk \\
\hline Swilk r1 \\
\hline \multicolumn{7}{|c|}{ Shapiro-Wilk W test for normal data } \\
\hline Variable & Obs & W & V & z & Prob >z \\
\hline R1 & 465 & 0,97148 & 8,988 & 5,261 & 0,00000 \\
\hline
\end{tabular}

Elaboración propia. 